\title{
A COMBUSTION WAVE OF PERMANENT FORM IN A COMPRESSIBLE GAS
}

\author{
LAWRENCE K. FORBES ${ }^{1}$ and WILLIAM DERRICK ${ }^{2}$
}

(Received 12 December, 1998; revised 2 February, 1999)

\begin{abstract}
A model is presented for describing the propagation of a one-dimensional wave of permanent form in a compressible gas in a pipe. Energy is lost to the system through the walls of the pipe, but the combustion wave produces heat through an exothermic chemical reaction. The full set of equations for the model is reduced to a phase-plane system, and it is shown that, for small amplitude waves, a weakly non-linear analysis leads to a temperature profile that is a classical solitary wave. A novel shooting method is developed for the full non-linear problem, and this confirms and extends the solitary-wave solution, up to a value of the temperature amplitude at which the wave begins to develop a shock. The jump conditions across the shock are presented, and numerical integration is used to continue the solution for temperature amplitudes at which a shock is present. An example is given of the extreme situation in which the shock is so strong that all the fuel behind the shock is exhausted.
\end{abstract}

\section{Introduction}

In recent years, a great deal of work has been devoted to the study of chemical reactions in which heat is produced, and in which at least some of the chemical conversion rates are highly sensitive to temperature. Thus, in addition to balance equations for the amounts of the various chemical species present, it is also necessary to include an energy balance equation, and for a well-mixed chemical reactor, the total system is therefore described by a system of ordinary differential equations. These are usually highly non-linear, in part because of the interaction terms between chemical components in the reactions, but more importantly because of the very non-linear nature of the dependence of the rates of the reactions upon the temperature. These non-linear differential equations have recently been shown to predict some very exotic

\footnotetext{
'School of Mathematics and Physics, The University of Tasmania, Hobart TAS 7001, Australia.

${ }^{2}$ Department of Mathematics, University of Montana, Missoula, Montana 59812, USA.

(C) Australian Mathematical Society 2001, Serial-fee code 0334-2700/01
} 
behaviour, such as periodic oscillations in the temperature, and even quasi-periodicity and chaos in some circumstances.

A simple model chemical reaction with heat production (exothermicity) and temperature sensitive rates was presented and analyzed by the Russian scientist Sal'nikov [20] in 1949. He envisaged a simple first-order reaction in which some initial species $C$ decomposed to form an intermediate substance $X$ which further decayed to form a final inert product $P$. The progress of this reaction is described mathematically by a rate equation for the concentration of the intermediate chemical $X$. However, since the rates are temperature sensitive and the reaction is exothermic, a second equation is needed to describe the energy balance, and because of this, the system is described by the concentration $[X]$ and the temperature $T$. Sal'nikov showed that his model reaction could exhibit multiple steady states, and was capable of producing timedependent oscillatory behaviour (limit cycles), since the steady states could undergo Hopf bifurcations.

Sal'nikov's system was analyzed by Gray and Roberts [11], using the modern methods of bifurcation and singularity theory. A separate analysis was undertaken by Kay and Scott [16] at about the same time, and in each case, the presence of oscillations in the temperature was confirmed. Later, Forbes [5] presented a numerical solution of the governing system of equations, using a shooting method in the phase plane, and obtained highly accurate results. For some values of the governing parameters, there are two concentric limit cycles in the phase plane, one of which is stable and the other unstable, and both of these could be obtained by Forbes' shooting method. A more complicated Sal'nikov reaction scheme (in which both of the first-order decay reactions had temperature sensitive rates) was later studied by Forbes, Myerscough and Gray [9] and this work showed a complicated range of behaviour in the phase plane, including multiple oscillations and homoclinic bifurcations. A subsequent analysis of this same scheme by Gray and Forbes [10] revealed an extraordinary degree of complexity in the behaviour of solutions to this problem.

It is, perhaps, not fully clear to what extent the exotic behaviour predicted by these models is actually observed experimentally. Nevertheless, oscillatory behaviour in the temperature is well-known (see Griffiths [15]), and Gray and Griffiths [12] have demonstrated that the Sal'nikov scheme can describe quite credibly the behaviour of an experimentally observed reaction system. Recently, Coppersthwaite, Griffiths and Gray [4] have shown that the model reaction is useful in describing the hydrogenchlorine reaction, and they argue that the oscillatory behaviour predicted by the mathematical model has practical safety ramifications in identifying parameter values that result in reactor instability and the possibility of explosion.

When the reaction is no longer well stirred, then spatial patterns can form in addition to the time dependent behaviour previously discussed, and the overall solutions can therefore become very complex indeed. At the other extreme, it is possible for 
purely spatial variations to occur that are independent of time, and these have been investigated for the Sal'nikov reaction in one dimension by Gray and Scott [14] and Forbes [6], and in the case of stationary axi-symmetric patterns by Forbes [7], for example.

In addition, it is possible for combustion systems such as Sal'nikov's reaction to exhibit travelling waves of permanent form. This intriguing possibility was apparently first recognized by Fisher and Kolmogorov in the context of biological systems (see Murray [18]), and this type of spatio-temporal pattern has since been widely studied in other situations. A simple model of a travelling combustion wave for an exothermic chemical system was proposed by Gray and Kordylewski [13], and involved only a single non-linear partial differential equation that expressed a balance between the diffusion and the generation of heat. This model has been extended by Tam [21] to incorporate the effects of the consumption of a solid fuel.

Recently, a similar system of two equations has been studied by Weber, Mercer, Sidhu and Gray [22]. Their model also balances thermal diffusion with exothermic heat production, and includes the effects of fuel consumption and diffusion. They carried out a detailed asymptotic and numerical analysis of their model, and showed the presence of travelling waves in addition to more exotic pulsing travelling waves that could undergo period doubling, possibly leading to spatio-temporal chaos. They set the ambient temperature equal to zero in their analysis, so as to avoid the 'cold boundary' problem associated with their choice of temperature dependent rate function; we discuss this further in Section 2. By varying a single parameter (the Lewis number), they claimed a wide applicability of their model, from gaseous fuels at one extreme through to solid fuels at the other. Models of this type have also been invoked as a means of describing the progress of large-scale bushfires, although Forbes [8] has recently questioned the applicability of travelling waves of permanent form to this situation.

It seems unlikely that a single model can account fully for combustion waves in both solid and gaseous fuels, although the equations proposed by Weber et al. [22] clearly provide a first approximation. It has long been known that the description of large amplitude travelling waves in gases requires that the chemical rate equations be coupled with the hydrodynamic equations of compressible flow, and this forms the basis of gaseous combustion and detonation theory. Much of this work is reviewed by Buckmaster [2], for example. Strong detonations in gases produce shock waves, and these must be described using the correct conservation conditions such as those discussed in Chapter 6 of the book by Williams [24]. By contrast, combustion waves in solids most likely occur as a gaseous intermediate species diffuses through the solid, and a model of this type has been proposed by Norbury and Stuart [19].

The purpose of the present paper is to examine combustion waves in a compressible gas. A travelling wave solution will be assumed, but the full equations of compress- 
ible gas flow will be included. This model therefore differs from that of Gray and Kordylewski [13], for example, in that it does not seek to balance chemical heat generation against thermal diffusion, but rather against the combined effects of thermal cooling to ambient and convective mass and momentum transfer. Support for this approach (at moderate and high Mach numbers) is given by Clarke [3], who showed that diffusive effects are weak in these cases. The 'cold boundary' problem is dealt with here, not by assuming an ambient temperature of absolute zero, but instead by employing an ignition temperature in the model for the temperature dependent rate function.

\section{The basic model}

We consider an infinitely long pipe extending along the $x$-axis, and initially imagine it to contain a mixture of two gaseous species which will be denoted as $A$ and $X$. The chemical $X$ will be thought of as the 'fuel', while species $A$ plays the role of oxygen in the combustion reaction

$$
A+X \stackrel{k(T)}{\longrightarrow} P \text {. }
$$

The product $P$ is taken to be an inert gas, and the rate of the reaction is given by the temperature sensitive function $k(T)$, where $T$ denotes temperature.

If brackets [-] denote concentration (moles per volume), then the reaction (2.1) leads to the three species conservation equations

$$
\begin{aligned}
& \frac{\partial[X]}{\partial t}+\frac{\partial}{\partial x}(u[X])=-[A][X] k(T) \\
& \frac{\partial[A]}{\partial t}+\frac{\partial}{\partial x}(u[A])=-[A][X] k(T) \\
& \frac{\partial[P]}{\partial t}+\frac{\partial}{\partial x}(u[P])=[A][X] k(T) .
\end{aligned}
$$

In these equations, $u$ represents the speed of the total gas in the positive $x$-direction, and $t$ is the time. This system (2.2) employs a similar approach to that in the paper by $\mathrm{Bi}$ and Agrawal [1]. Suppose that the molecular weights (mass per mole) of the three chemical species $X, A$ and $P$ are $m_{X}, m_{A}$ and $m_{P}$ respectively. Then the density $\rho$ of the gas in the pipe (mass per volume) is given by the relation

$$
\rho=m_{X}[X]+m_{A}[A]+m_{P}[P],
$$

and the stoichiometry of the reaction (2.1) requires in addition that

$$
m_{P}=m_{X}+m_{A} .
$$


It is necessary now to consider the fluid mechanics of the combustion wave. Conservation of mass for a compressible gas gives rise to the usual continuity equation

$$
\frac{\partial \rho}{\partial t}+\frac{\partial(\rho u)}{\partial x}=0
$$

In fact, this condition (2.5) can be derived from the three species equations (2.2) by multiplying each by the appropriate molecular weight for that species and adding, making use of (2.3) and (2.4). The momentum conservation equation is also well known, and is expressed by the compressible Euler equation

$$
\frac{\partial}{\partial t}(\rho u)+\frac{\partial}{\partial x}\left(p+\rho u^{2}\right)=0
$$

in the absence of viscosity and ignoring body forces. Here, $p$ is the gas pressure, which from the ideal gas law, is related to the density and temperature by the relation

$$
p=\rho R T,
$$

in which $R$ is the gas constant.

The final equation in the system expresses the energy balance, and is the most complicated. By making use of the relation on page 186 of Liepmann and Roshko [17] there results, after considerable algebra, the equation

$$
\begin{aligned}
& \frac{\partial}{\partial t}\left(\frac{\gamma R T}{\gamma-1}+\frac{1}{2} u^{2}\right)+u \frac{\partial}{\partial x}\left(\frac{\gamma R T}{\gamma-1}+\frac{1}{2} u^{2}\right) \\
& \quad=\frac{1}{\rho}\left[-N\left(T-T_{a}\right)+Q_{X}[X][A] k(T)+\frac{\partial p}{\partial t}\right] .
\end{aligned}
$$

The left-hand side of this expression represents the convective rate of change of enthalpy, and the first term on the right-hand side is the energy loss to ambient; here, $T_{a}$ is the ambient temperature and $N$ is a heat loss coefficient. The second term on the right represents the heat production by the exothermic reaction $(2.1)$, and the coefficient $Q_{X}$ is a constant. The constant $\gamma$ is the ratio of specific heats, and $k(T)$ is the reaction rate, as before.

Dimensionless variables are now introduced into the problem. In order to do this, it is necessary to consider the rate function $k(T)$, which if Arrhenius kinetics were assumed, would have the form

$$
k(T)=\text { constant } \times \exp (-E / R T),
$$

in which the constant $E$ is an activation energy for the reaction. In this paper, we do not use this pure Arrhenius function, but the activation energy $E$ is nevertheless an 
important concept for the reaction, and we use this quantity to define a dimensionless temperature and flow speed

$$
\hat{T}=R T / E \quad \text { and } \quad \hat{u}=u / \sqrt{E} .
$$

If $L$ represents some characteristic length scale in the problem, then it is natural to define dimensionless space and time coordinates to be

$$
\hat{x}=x / L \quad \text { and } \quad \hat{t}=t \sqrt{E} / L .
$$

The appropriate non-dimensional density and pressure functions then become

$$
\hat{\rho}=\rho R \sqrt{E} /(N L) \quad \text { and } \quad \hat{p}=p R /(N L \sqrt{E}),
$$

and the dimensionless gas concentrations and rate function become

$$
\hat{X}=[X] \frac{R Q_{X}}{N L \sqrt{E}}, \quad \hat{A}=[A] \frac{R Q_{X}}{N L \sqrt{E}} \quad \text { and } \quad \hat{k}(\hat{T})=k(T) \frac{N L^{2}}{R Q_{X}} .
$$

In the rest of this paper, only non-dimensional variables will be used, and so the superscript ^ will be dropped henceforth. In these new dimensionless variables, the mass continuity equation and the momentum equation have the same forms as (2.5) and (2.6), and the energy equation (2.8) becomes

$$
\begin{gathered}
\frac{\partial}{\partial t}\left(\frac{\gamma T}{\gamma-1}+\frac{1}{2} u^{2}\right)+u \frac{\partial}{\partial x}\left(\frac{\gamma T}{\gamma-1}+\frac{1}{2} u^{2}\right) \\
=\frac{1}{\rho}\left[-\left(T-\theta_{a}\right)+A X k(T)+\frac{\partial p}{\partial t}\right] .
\end{gathered}
$$

Here, the constant $\theta_{a}=R T_{a} / E$ is the non-dimensional ambient temperature. The first of the equations in the system (2.2) takes the dimensionless form

$$
\frac{\partial X}{\partial t}+\frac{\partial}{\partial x}(u X)=-A X k(T),
$$

and subtracting the first two equations in the system (2.2) yields

$$
\frac{\partial}{\partial t}(X-A)+\frac{\partial}{\partial x}(u(X-A))=0 .
$$

This is equivalent to eliminating the concentration of the product $P$ from the third equation in (2.2), using (2.3) and (2.4). Finally, the gas law (2.7) becomes simply

$$
p=\rho T
$$

in dimensionless form. 
It is necessary now to consider an appropriate form for the rate function $k(T)$. This is important because it is clear that the use of a straightforward function such as the Arrhenius model (2.9) will lead to some sort of 'cold boundary' difficulty, in which the burning reaction (2.1) will proceed at some finite rate for all temperatures above absolute zero. Weber et al. [22] avoided this problem by setting the ambient temperature $\theta_{a}$ to zero in their analysis, and justified this by arguing that the ambient temperature is such a small fraction of the maximum that it can be ignored, particularly as the Arrhenius rate (2.9) is exponentially small at those temperatures.

Our approach is different to this, and is more in keeping with Williams' [24] use of an ignition temperature, below which the rate is taken to be zero. The Arrhenius function (2.9) itself must only be an approximate representation of the true situation at room temperatures, and in this paper we replace it with the function

$$
k(T)= \begin{cases}\beta\left[1-\exp \left(\theta_{a}-T\right)\right], & \text { if } T>\theta_{a} \\ 0, & \text { if } T<\theta_{a}\end{cases}
$$

This function has all the qualitative features of the Arrhenius model, since it has a finite limiting value as $T \rightarrow \infty$, and it is zero for $T=0$. In addition, a straight line of appropriate slope could intersect the graph of the function (2.14) in three different places, showing that the function is capable of generating the three steadystate values that typify combustion in a well-mixed system with Newtonian cooling to ambient. However, we believe that the model rate (2.14) has the advantage over the Arrhenius function (2.9) that it avoids the 'cold boundary' difficulty at finite ambient temperature $\theta_{a}$ and, although it is true that the Arrhenius rate becomes exponentially small for small temperatures, the nett energy loss over an infinitely long pipe may not necessarily be small. Clearly other functions of the type (2.14) could be used for the dependence of rate upon temperature, and (2.14) has been chosen simply to minimize the number of dimensionless parameters in this problem, since the ignition temperature has been assumed to be equal to the ambient temperature $\theta_{a}$. In a model of bushfire spread, Forbes [8] employed a 'shifted' Arrhenius function that had similar qualitative properties to (2.14).

Solutions to this problem are therefore characterized by three dimensionless parameters: $\gamma$, which is the ratio of specific heats and will be taken to have the value 1.4 in this paper, $\theta_{a}=R T_{a} / E$ the ambient temperature, and the rate constant $\beta$ that appears in (2.14). In addition, it is necessary to specify the quiescent conditions ahead of the combustion wave, and this introduces two extra parameters. In these dimensionless variables, it is assumed that

$$
\begin{aligned}
& A \rightarrow 1, \quad X \rightarrow X_{0}, \quad u \rightarrow 0, \\
& T \rightarrow \theta_{a}, \quad \rho \rightarrow \rho_{0}, \quad p \rightarrow \rho_{0} \theta_{a}, \quad \text { as } x \rightarrow \infty
\end{aligned}
$$


for a wave travelling from left to right. To the extent that the length scale $L$ used to create the dimensionless coordinate $x$ has not been specified, the ambient density $\rho_{0}$ ahead of the wave can be set to one without loss of generality, and this is assumed from now on. However, the parameter $X_{0}$, which is the initial fuel concentration, is the fourth dimensionless quantity that can be varied independently.

\section{Reduction to a phase-plane system for waves of moderate amplitude}

We are concerned with travelling-wave solutions to the dimensionless system of six non-linear partial differential equations (2.5), (2.6) and (2.10)-(2.13). A coordinate

$$
\xi=x-c t
$$

moving with the wave is introduced, where now the wave speed $c$ is a constant eigenvalue that must be determined.

The mass equation (2.5) immediately becomes

$$
-c \rho^{\prime}+(\rho u)^{\prime}=0
$$

and the momentum conservation law (2.6) gives

$$
-c(\rho u)^{\prime}+\left(\rho u^{2}+p\right)^{\prime}=0 .
$$

The energy condition (2.10) reduces to the equation

$$
(-c+u)\left(\frac{\gamma T}{\gamma-1}+\frac{1}{2} u^{2}\right)^{\prime}=\frac{1}{\rho}\left[-\left(T-\theta_{a}\right)+A X k(T)-c p^{\prime}\right] .
$$

In the moving coordinate $\xi$, the rate law (2.11) takes the form

$$
-c X^{\prime}+(u X)^{\prime}=-A X k(T),
$$

and the species equation (2.12) becomes

$$
-c(X-A)^{\prime}+(u(X-A))^{\prime}=0 .
$$

The remaining equation is the gas law (2.13) which is unchanged by adopting the new moving coordinate system.

Equation (3.1) can be integrated immediately. After making use of the upstream conditions (2.15), the result is

$$
\rho=c \rho_{0} /(c-u),
$$

which effectively eliminates the density variable $\rho$ in favour of the gas speed $u$. The reduced momentum equation (3.2) can also be integrated, and when combined with 
the upstream conditions (2.15) and (3.6), it gives a formula for the gas pressure $p$ which may be expressed as

$$
p=\rho_{0}\left[\theta_{a}+c u\right] .
$$

Equations (3.6) and (3.7) may now be substituted into the gas law (2.13) and solved for the temperature $T$. This gives

$$
T=\theta_{a}+u\left(c-\theta_{a} / c\right)-u^{2} .
$$

Finally, the species equation (3.5) can also be integrated to give the relation

$$
A=X-\frac{c\left(X_{0}-1\right)}{c-u}
$$

between the concentrations of the two gaseous species $\mathrm{X}$ and $\mathrm{A}$, in the wave.

Equations (3.6)-(3.9) effectively eliminate the density $\rho$, pressure $p$, temperature $T$ and oxygen concentration $A$, in favour of the gas speed $u$ and the fuel concentration $X$. The two remaining equations (3.3) and (3.4) then become a system simply in the $(u, X)$ phase plane. When combined with (3.6)-(3.8), the energy equation (3.3) gives

$$
u^{\prime}=\frac{(\gamma-1)\left[T-\theta_{a}-A X k(T)\right]}{\rho_{0}\left[c^{2}-\gamma \theta_{a}-c(\gamma+1) u\right]}
$$

and the rate law (3.4) can be written

$$
X^{\prime}=\frac{u^{\prime} X+A X k(T)}{c-u} .
$$

The original system has thus been reduced essentially to the two equations ( 3.10$)$ and (3.11), for a travelling wave of permanent form.

\section{The weakly non-linear analysis}

In this section, an approximate solution is developed for the system of equations (3.6)-(3.11) above. This will be seen to give very good agreement with the full nonlinear solution, for waves of small to medium amplitude. Additionally, it provides vital guidance as to how the numerical shooting method of Section 5 should be structured, so as to give a well-posed system capable of great accuracy.

We introduce the perturbation scheme

$$
\begin{aligned}
u(\xi) & =\quad \epsilon U_{1}(\xi)+\mathscr{O}\left(\epsilon^{2}\right), \\
X(\xi) & =X_{0}+\epsilon X_{1}(\xi)+\mathscr{O}\left(\epsilon^{2}\right), \\
c & =\sqrt{\theta_{a}}+\epsilon c_{1}+\mathscr{O}\left(\epsilon^{2}\right),
\end{aligned}
$$


in which $\epsilon$ is some small parameter, as yet unspecified.

When the expansion (4.1) is inserted into (3.6), the density function becomes

$$
\rho=\rho_{0}\left[1+\epsilon \frac{U_{1}}{\sqrt{\theta_{a}}}+\mathscr{O}\left(\epsilon^{2}\right)\right]
$$

and (3.7) gives the pressure, to first order, in the form

$$
p=\rho_{0}\left[\theta_{a}+\epsilon \sqrt{\theta_{a}} U_{1}+\mathscr{O}\left(\epsilon^{2}\right)\right] .
$$

After some algebra, it is possible to show from (3.8) and (4.1) that the temperature in the wave can be expressed as

$$
T=\theta_{a}+\epsilon^{2}\left(2 c_{1} U_{1}-U_{1}^{2}\right)+\mathscr{O}\left(\epsilon^{3}\right) .
$$

This equation (4.4) is interesting, since it shows that the perturbations to temperature are second order in $\epsilon$, whereas those for all the other variables are first order. Finally, the equation (3.9) for oxygen concentration yields

$$
A=1+\epsilon\left[X_{1}-\frac{\left(X_{0}-1\right) U_{1}}{\sqrt{\theta_{a}}}\right]+\mathscr{O}\left(\epsilon^{2}\right) .
$$

It is also necessary to expand the rate function (2.14) as a series in $\epsilon$. This is done using (4.4), and for $T>\theta_{a}$ gives

$$
k(T)=\epsilon^{2} \beta\left(2 c_{1} U_{1}-U_{1}^{2}\right)+\mathscr{O}\left(\epsilon^{3}\right) .
$$

In order to continue the perturbation expansions, and apply them to the phase-plane equations (3.10) and (3.11), it becomes clear that the travelling wave coordinate $\xi$ must be stretched. To this end, a new coordinate $\eta$ is introduced, and is defined through the relation

$$
\xi=\eta / \epsilon .
$$

This scaling is a consequence of the second-order nature of the perturbations in (4.4) and (4.6). The energy equation (3.10) thus yields the condition

$$
\frac{d U_{1}}{d \eta}=-\Gamma_{1}\left(2 c_{1} U_{1}-U_{1}^{2}\right)
$$

at second order in $\epsilon$, and the rate equation (3.11) results in

$$
\frac{d X_{1}}{d \eta}=\Delta_{1}\left(2 c_{1} U_{1}-U_{1}^{2}\right)
$$


Here, for simplicity, we have defined the two constants

$$
\Gamma_{1}=\frac{1-\beta X_{0}}{\rho_{0} \theta_{a}}, \quad \Delta_{1}=\frac{X_{0}}{\sqrt{\theta_{a}}}\left(\beta-\Gamma_{1}\right) .
$$

The condition (4.7) is a straightforward logistic equation for the perturbed velocity function $U_{1}$. However, its solution only satisfies the boundary condition $U_{1} \rightarrow 0$ as $\eta \rightarrow \infty$ (which comes from (2.15)) if the restriction $\Gamma_{1}>0$ is imposed. In addition, there is a single arbitrary constant in the general solution to the first-order differential equation (4.7), and this is determined from the direction field by adding the requirement that at $\eta=0$, the perturbed gas speed $U_{1}$ has half its value far downstream. (This imposes no loss of generality, and merely defines the location of the origin of $\eta$.) Thus we set $U_{1}=c_{1}$ at $\eta=0$, and obtain the solution for $U_{1}$ in the final form

$$
U_{1}=\frac{2 c_{1} \exp \left(-2 c_{1} \Gamma_{1} \eta\right)}{1+\exp \left(-2 c_{1} \Gamma_{1} \eta\right)}
$$

As yet, the constant $c_{1}$ is unknown.

Equation (4.8) involves a simple quadrature for the perturbation function $X_{1}$ to the fuel concentration. This involves an integration constant, which must be determined by imposing the condition $X_{1} \rightarrow 0$ as $\eta \rightarrow \infty$ (which comes from the boundary conditions (2.15)). This gives the solution

$$
X_{1}=-\frac{\Delta_{1}}{\Gamma_{1}} \frac{2 c_{1} \exp \left(-2 c_{1} \Gamma_{1} \eta\right)}{1+\exp \left(-2 c_{1} \Gamma_{1} \eta\right)}
$$

where the constants $\Gamma_{1}$ and $\Delta_{1}$ are as defined in (4.9).

The other variables can now be obtained in a straightforward manner from these solution functions (4.10) and (4.11), by making use of (4.2)-(4.5). Of particular interest is the temperature which, from (4.4), involved second-order perturbation terms. The solution (4.10) shows that the temperature has the weakly non-linear form

$$
T=\theta_{a}+\epsilon^{2} c_{1}^{2} \operatorname{sech}^{2}\left(c_{1} \Gamma_{1} \eta\right)+\mathscr{O}\left(\epsilon^{3}\right) .
$$

Thus the temperature in a small amplitude wave takes precisely the form of a travelling soliton. If the temperature amplitude $\mathscr{A}_{T}$ of the wave is specified in advance, and defined to be the difference between the maximum and minimum values of temperature, then it follows from (4.12) that $\mathscr{A}_{T}=\epsilon^{2} c_{1}^{2}$, and so the wave speed is found from (4.1) to be

$$
c=\sqrt{\theta_{a}}+\sqrt{\mathscr{A}_{T}}+\mathscr{O}\left(\epsilon^{2}\right) .
$$




\section{The shooting method for moderate amplitude waves}

The approximate solution of the preceding section provides a valuable guide in designing an efficient, stable and accurate numerical shooting method for solving the full non-linear system of equations (3.6)-(3.11). To begin, some numerical window $-K<\xi<K$ about the moving wave is discretized using equally spaced grid points $\xi_{1}, \ldots, \xi_{\text {mid }}, \ldots, \xi_{2 M+1}$. The $2 M+1$ grid points are separated by the interval $\Delta \xi$, and chosen so that $\xi_{1}=-K, \xi_{\text {mid }}=0$ and $\xi_{2 M+1}=K$.

Because of the loss term (to ambient) in the energy equation (2.10), the temperature far behind the wave $(\xi \rightarrow-\infty)$ must ultimately return to the ambient value $\theta_{a}$. From (3.8), the gas speed far downstream must then be $u \rightarrow c-\theta_{a} / c$. In obtaining the approximate solution (4.10), it was necessary to impose the condition that the speed $u_{\text {mid }}$ at $\xi=0$ should have half its final downstream value. We follow this in the full non-linear solution, and thus require that

$$
u_{\text {mid }}=\left(c-\theta_{a} / c\right) / 2 \text { at } \xi_{\text {mid }}=0 \text {. }
$$

In addition, following the development leading to (4.13), a temperature amplitude $\mathscr{A}_{T}$ for the wave is specified in advance, and the solution is required to satisfy the condition

$$
T_{\text {mid }}=\theta_{a}+\mathscr{A}_{T} \quad \text { at } \xi_{\text {mid }}=0
$$

In essence, the condition (5.2) merely defines the origin of the $\xi$ coordinate.

When conditions (5.1) and (5.2) are substituted into the temperature equation (3.8) at $\xi=0$, an explicit formula for the wave speed $c$ is obtained. The result is

$$
c=\sqrt{\mathscr{A}_{T}}+\sqrt{\mathscr{A}_{T}+\theta_{a}}, \quad u_{\text {mid }}=\sqrt{\mathscr{A}_{T}} .
$$

The first order formula (4.13) is evidently the weakly non-linear approximation to the exact equation (5.3) for the wave speed $c$.

A remarkably simple shooting method can now be developed for solving the full system of non-linear equations. The wave amplitude $\mathscr{A}_{T}$ is specified, and the wave speed is computed according to the formula in (5.3). Now in order to obtain the approximate solution (4.11) for the fuel concentration $X$, it was necessary to specify an additive constant of integration so as to satisfy the upstream boundary condition (2.15). This same approach is needed here, for the fully non-linear problem. Thus we make an initial guess at the value $X_{\text {mid }}$ of the fuel concentration at the mid-point $\xi=0$, and on the basis of this guess, the ordinary differential equations (3.10) and (3.11) are integrated both upstream to the point $\xi=K$ and downstream to $\xi=-K$. Since the fuel concentration must achieve its ambient value far upstream, we form 
the residual error function $E_{R}=X_{2 M+1}-X_{0}$ and use Newton's method to vary the midpoint value $X_{\text {mid }}$ until $E_{R}$ has been made arbitrarily close to zero. The standard fourth-order Runge-Kutta formula is used to integrate the differential equations (3.10) and (3.11), and it is found that the method converges in 5 or 6 iterations. To start the iteration for very small amplitudes, an initial guess based on the approximate solution (4.11) may be used in the form

$$
X_{\text {mid }} \approx X_{0}-\sqrt{\mathscr{A}_{T}} \Delta_{1} / \Gamma_{1},
$$

and for higher values of the temperature amplitude, a previously computed solution for a lower amplitude provides a good starting value.

\section{Numerical results for moderate amplitude waves}

The numerical method of Section 5 has been run for a reasonably large set of parameter values, and produces highly accurate results extremely rapidly. We have run the shooting method code with as many as one million points either side of the midpoint, so that the total number of grid points $2 M+1$ is of the order of two million. Converged results are obtained in about two minutes of computing time on a SPARC ULTRA 1 computer, and with this number of mesh points, may be considered to be essentially exact. This is therefore a remarkably good numerical scheme, at least for this travelling wave problem.

Some representative solutions are shown in Figures 1. Here, we have set the reference reaction rate $\beta=1$ and the ambient concentration $X_{0}=1$, and chosen an ambient temperature $\theta_{a}=0.25$. These values satisfy the condition $\Gamma_{1}>0$ as required for the weakly non-linear solution of Section 4 , where $\Gamma_{1}$ is defined as in (4.9). Three sets of results are shown, for temperature amplitudes $\mathscr{A}_{T}=0.002$ (drawn with a dotted line), 0.004 (chain-dot line) and 0.0071 (solid line). We note that, for $X_{0}=1$, the oxygen concentration $A$ is the same as the fuel concentration $X$ by (3.9), and so there is no need in this case to display solutions for this variable.

The gas speed $u$ as a function of position $\xi$ along the travelling wave is shown in Figure 1(a). Far ahead of the wave, conditions are quiescent, so that $u \rightarrow 0$ as $\xi \rightarrow \infty$. There is a reasonably rapid rise in the velocity at the wave front, and far downstream the gas velocity has the asymptotic value $u \rightarrow c-\theta_{a} / c$ as $\xi \rightarrow-\infty$, which follows from (3.8) (see the discussion leading up to (5.1)). For small temperature amplitudes, these wave profiles are in excellent agreement with the asymptotic solution (4.10), but differences begin to appear as the amplitude $\mathscr{A}_{T}$ is increased. Specifically, the wave front steepens with increasing amplitude, until the numerical method fails at about $\mathscr{A}_{T}=0.0071$. It is natural to suspect this may be due to the formation of a shock in the wave profile at about this temperature amplitude, and this is indeed the case; shocks will be considered in the next two sections. 


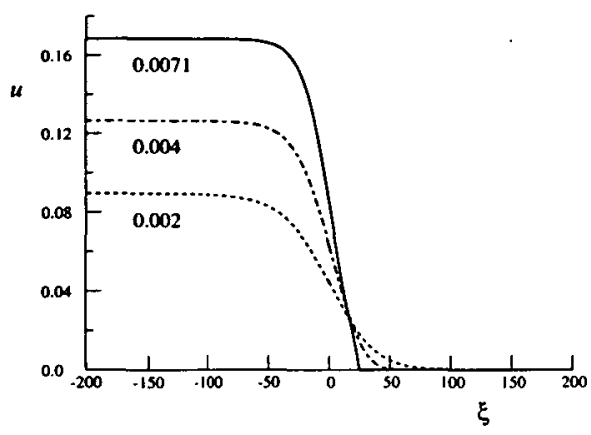

(a)

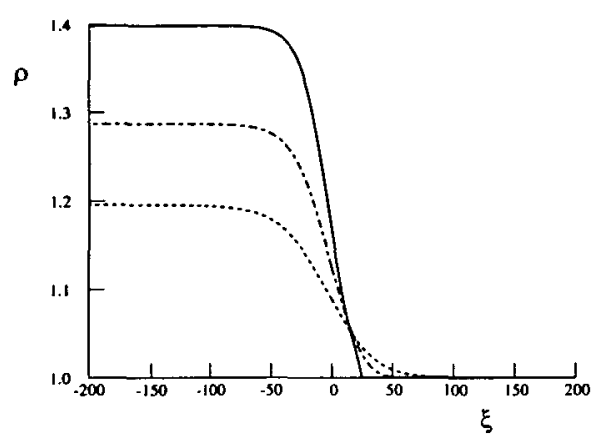

(c)

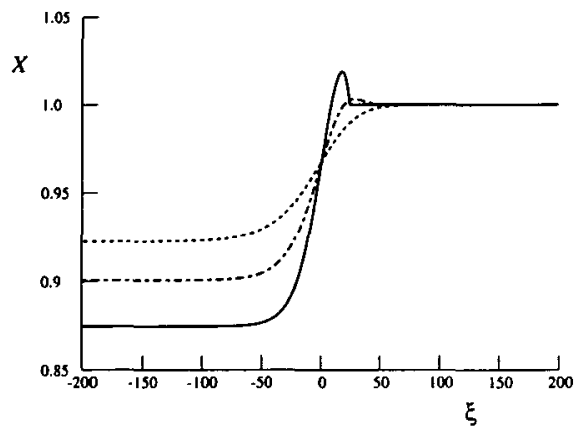

(b)

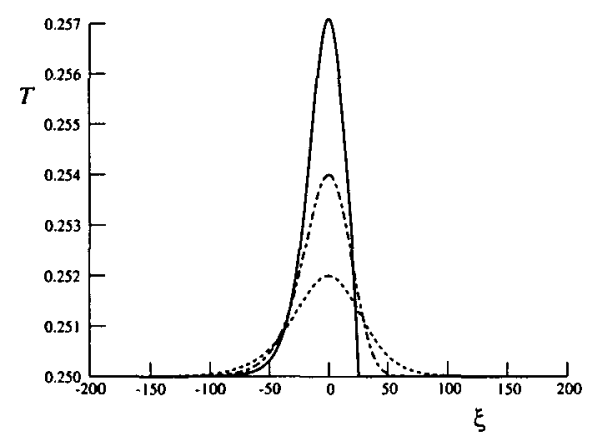

(d)

FIGURE 1. Wave profiles for $\beta=0.9, X_{0}=1$ and $\theta_{a}=0.25$, for the three values of total wave amplitude $\mathscr{A}_{T}=0.002$ (dotted line), 0.004 (chain-dot line) and 0.0071 (solid line). Profiles shown are for (a) gas velocity, (b) fuel concentration, (c) gas density and (d) gas temperature.

Figure 1(b) shows the fuel concentration $X$ in the wave. For small wave amplitudes $\mathscr{A}_{T}$ the solutions agree well with the weakly non-linear result in (4.11), but the profile deviates from this solution as the amplitude is increased, as is to be expected. There is steepening of the wave front as in Figure 1(a), and interestingly, the concentration $X$ actually increases above its quiescent value $X_{0}=1$ immediately before the shock. This is possible because the gas in the pipe is compressible.

This compressibility effect is evident from Figure 1(c), in which the gas density $\rho$ is plotted against $\xi$, along the wave. The moving combustion wave has the effect of compressing the gas behind it, so that there is a permanent rise in the density, as is evident from the diagram.

Finally, the temperature profile along the wave is displayed in Figure 1(d), for the three different wave amplitudes indicated. The smallest amplitude $\mathscr{A}_{T}=0.002$ gives a solution that is very close to the classical soliton, given by (4.12), and as expected, this profile is very nearly symmetrical about $\xi=0$. The symmetry is lost as the 
amplitude increases, however, since the wave steepens at the front, and for the largest amplitude $\mathscr{A}_{T}=0.0071$ shown in the diagram, there is a near vertical section at the very front of the profile. This case is very near to the temperature amplitude at which a shock forms, and this effect must now be considered separately.

\section{Inclusion of a shock in the model}

It is evident from the numerical results in Section 6 that shocks are a possibility in this model (because of the neglect of thermal diffusion and viscosity) and so it is necessary now to develop the jump conditions for the variables across the shock. These may be found in the book by Whitham [23] (see page 40, for example). For a general hyperbolic conservation law of the form

$$
\frac{\partial \mathbf{U}}{\partial t}+\frac{\partial \mathbf{F}}{\partial x}=\mathbf{0}
$$

the appropriate weak solution permits jumps which satisfy the discontinuity condition

$$
c_{s}\left(\mathbf{U}_{2}-\mathbf{U}_{1}\right)=\left(\mathbf{F}_{2}-\mathbf{F}_{1}\right),
$$

where the subscripts 1 and 2 refer to conditions either side of the shock, which moves with shock speed $c_{s}$. Here, subscript 1 will be taken to refer to conditions ahead of the wave, and 2 will denote the values of variables behind the wave.

All the governing partial differential equations in Section 2 are already expressed in the conservation form (7.1), except the energy condition (2.10). This is combined with the mass condition (2.5) to give

$$
\begin{gathered}
\frac{\partial}{\partial t}\left(\rho\left[\frac{\gamma T}{\gamma-1}+\frac{u^{2}}{2}\right]-p\right)+\frac{\partial}{\partial x}\left(\rho u\left[\frac{\gamma T}{\gamma-1}+\frac{u^{2}}{2}\right]\right) \\
=-\left(T-\theta_{a}\right)+A X k(T) .
\end{gathered}
$$

The jump conditions (7.2), when applied to the continuity equation (2.5), give the mass shock condition

$$
c_{s}\left(\rho_{2}-\rho_{1}\right)=\rho_{2} u_{2}-\rho_{1} u_{1},
$$

and the momentum equation (2.6) yields

$$
c_{s}\left(\rho_{2} u_{2}-\rho_{1} u_{1}\right)=\rho_{2} u_{2}^{2}+p_{2}-\rho_{1} u_{1}^{2}-p_{1} .
$$

The energy equation, written in the correct conservation form (7.3), gives the jump condition

$$
c_{s}\left(\rho_{2}\left[\frac{\gamma T_{2}}{\gamma-1}+\frac{u_{2}^{2}}{2}\right]-p_{2}-\rho_{1}\left[\frac{\gamma T_{1}}{\gamma-1}+\frac{u_{1}^{2}}{2}\right]+p_{1}\right)
$$




$$
=\rho_{2} u_{2}\left[\frac{\gamma T_{2}}{\gamma-1}+\frac{u_{2}^{2}}{2}\right]-\rho_{1} u_{1}\left[\frac{\gamma T_{1}}{\gamma-1}+\frac{u_{1}^{2}}{2}\right]
$$

across the shock. From the rate equation (2.11), we obtain

$$
c_{s}\left(X_{2}-X_{1}\right)=u_{2} X_{2}-u_{1} X_{1} \text {, }
$$

and the species equation (2.12) gives

$$
c_{s}\left(X_{2}-A_{2}-X_{1}+A_{1}\right)=u_{2}\left(X_{2}-A_{2}\right)-u_{1}\left(X_{1}-A_{1}\right) \text {. }
$$

On both sides of the shock, the ideal gas law (2.13) applies. The six equations (7.4) $-(7.8)$ and (2.13) involve the seven unknowns $c_{s}, \rho_{2}, u_{2}, p_{2}, T_{2}, X_{2}$ and $A_{2}$.

It is possible to define gas velocities relative to the shock to be

$$
\bar{u}_{1}=c_{s}-u_{1} \text { and } \quad \bar{u}_{2}=c_{s}-u_{2} \text {. }
$$

In terms of these quantities, the mass jump condition (7.4) becomes

$$
\rho_{2} \bar{u}_{2}=\rho_{1} \bar{u}_{1}
$$

the momentum equation (7.5) takes the form

$$
\rho_{2} \bar{u}_{2}^{2}+p_{2}=\rho_{1} \bar{u}_{1}^{2}+p_{1}
$$

and after some re-arrangement, the energy condition (7.6) can be written

$$
\frac{\gamma T_{2}}{\gamma-1}+\frac{\bar{u}_{2}^{2}}{2}=\frac{\gamma T_{1}}{\gamma-1}+\frac{\bar{u}_{1}^{2}}{2}
$$

These three equations (7.10)-(7.12) are essentially just the well-known RankineHugoniot conditions for a normal shock (see Liepmann and Roshko [17, page 56]), but in coordinates moving relative to the shock speed $c_{s}$. In terms of the relative velocities (7.9), the rate law (7.7) yields

$$
\bar{u}_{2} X_{2}=\bar{u}_{1} X_{1}
$$

and the species equation (7.8) becomes

$$
\bar{u}_{2}\left(X_{2}-A_{2}\right)=\bar{u}_{1}\left(X_{1}-A_{1}\right) .
$$

In order to solve these equations (7.9)-(7.14), we specify the shock temperature amplitude $\mathscr{A}_{s}=T_{2}-T_{1}$, so that the temperature $T_{2}$ immediately behind the shock is known. Eliminating $\bar{u}_{2}$ and then $\bar{u}_{1}^{2}$ gives

$$
\rho_{2}=\frac{\rho_{1} \Omega_{s}}{2\left(T_{1}+\mathscr{A}_{s}\right)}
$$


where

$$
\Omega_{s}=\left(\frac{\gamma+1}{\gamma-1}\right) \mathscr{A}_{s}+\left[\left(\frac{\gamma+1}{\gamma-1}\right)^{2} \mathscr{A}_{s}^{2}+4\left(T_{1}+\mathscr{A}_{s}\right) T_{1}\right]^{1 / 2} .
$$

The shock speed is found to be

$$
c_{s}=u_{1}+\Omega_{s}\left[\frac{\gamma}{(\gamma+1) \Omega_{s}-2(\gamma-1)\left(T_{1}+\mathscr{A}_{s}\right)}\right]^{1 / 2}
$$

and the gas speed behind the shock is

$$
u_{2}=c_{s}-\left(\rho_{1} / \rho_{2}\right)\left(c_{s}-u_{1}\right)
$$

where the shock speed is calculated from (7.16) and the density ratio from (7.15a). The pressure and temperature immediately behind the shock are now able to be determined in the form

$$
p_{2}=\rho_{1} \Omega_{s} / 2, \quad T_{2}=T_{1}+\mathscr{A}_{s},
$$

in which the quantity $\Omega_{s}$ is given by (7.15b). Finally, the fuel and oxygen concentration are computed to be

$$
X_{2}=\frac{X_{1} \Omega_{s}}{2\left(T_{1}+\mathscr{A}_{s}\right)}, \quad A_{2}=\frac{A_{1} \Omega_{s}}{2\left(T_{1}+\mathscr{A}_{s}\right)} .
$$

It is evident from the numerical results in Figures 1, for example, that the shock forms at the very foot of the wave front, where it is the first part of the combustion front that meets the quiescent upstream conditions. Thus, in (7.15)-(7.19), it is necessary to set $u_{1}=0, \rho_{1}=\rho_{0}, T_{1}=\theta_{a}, X_{1}=X_{0}$ and $A_{1}=1$ in order to determine the conditions immediately behind the shock. Then the phase plane equations in Section 3 may be integrated downstream from the shock to give the complete solution. The governing equations are the system (3.1)-(3.5) and the gas law (2.13), except that the wave propagation speed $c$ is now replaced by the shock speed $c_{s}$.

With a shock present in the wave front, (3.6) is replaced by

$$
\rho=\left(c_{s}-u_{2}\right) \rho_{2} /\left(c_{s}-u\right),
$$

to give the density downstream of the shock in terms of the gas speed $u$. The pressure and temperature, replacing (3.7) and (3.8) when a shock is present, are computed to be

$$
p=p_{2}+\rho_{2}\left(u-u_{2}\right)\left(c_{s}-u_{2}\right)
$$

and

$$
T=\frac{p_{2}\left(c_{s}-u\right)}{\rho_{2}\left(c_{s}-u_{2}\right)}+\left(u-u_{2}\right)\left(c_{s}-u\right)
$$


respectively, and the relation (3.9) for the oxygen concentration downstream of the shock is replaced with the expression

$$
A=X-\frac{\left(c_{s}-u_{2}\right)\left(X_{2}-A_{2}\right)}{c_{s}-u} .
$$

The first equation (3.10) in the phase plane system is replaced with the differential equation

$$
u^{\prime}=\frac{(\gamma-1)\left[T-\theta_{a}-A X k(T)\right]}{\rho_{2}\left[\left(c_{s}+\gamma u_{2}\right)\left(c_{s}-u_{2}\right)-\gamma T_{2}-u(1+\gamma)\left(c_{s}-u_{2}\right)\right]}
$$

and the second of the two phase plane equations is exactly the same as (3.11), except that the speed $c$ is replaced with $c_{s}$. The shock is taken to be located at the point $\xi=0$, and this system of two differential equations (7.24) and (3.11), with the subsidiary equations (7.20)-(7.23), is integrated downstream to the edge of the computational window at $\xi=-K$. In this way, the shock-fitted combustion wave is computed to very high accuracy, and there is no need here for the iterative shooting approach of Section 5 .

\section{Numerical results for shock propagation}

It is possible to calculate the precise value of the wave amplitude $\mathscr{A}_{T}$ at which the shock first forms. Setting the shock amplitude $\mathscr{A}_{s}$ to zero in (7.16) gives the speed of the shock of infinitesimal amplitude to be $c_{s} \rightarrow \sqrt{\gamma \theta_{a}}$; in dimensional variables, this is precisely the speed of sound at ambient temperature. When this is equated to the expression for the wave speed in (5.3), it is found that the shock first forms when

$$
\mathscr{A}_{T} \rightarrow \frac{(\gamma-1)^{2} \theta_{a}}{4 \gamma}
$$

With $\gamma=1.4$ and $\theta_{a}=0.25$ as in Section 6, this result (8.1) indicates that the shock first appears when $\mathscr{A}_{T}=0.00714825$ (with a speed $c_{s}=0.59161$ ) and this is in very good agreement with Figures 1.

The results shown in Figures 2 continue those presented in Figures 1, beyond the point at which the shock has formed. The reference reaction rate is $\beta=0.9$, the fuel concentration far upstream is $X_{0}=1$ and the ambient temperature has the dimensionless value $\theta_{a}=0.25$, as before. Wave profiles are shown for three different wave amplitudes. The smallest has shock amplitude $\mathscr{A}_{s}=0.003$ and is drawn with a dotted line, the profile of intermediate height, drawn with a chain-dot line, has shock amplitude 0.006 and the largest amplitude profile has $\mathscr{A}_{s}=0.015$ and is indicated with 


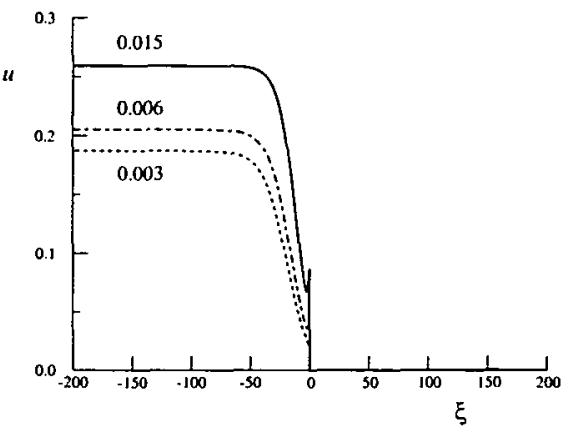

(a)

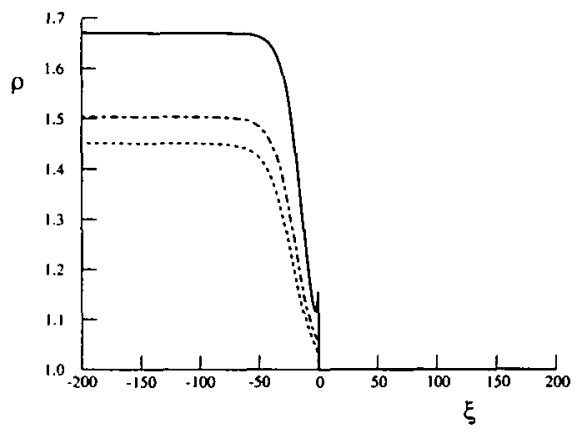

(c)

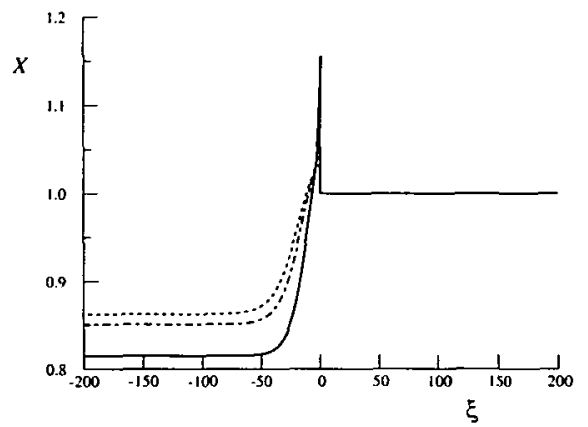

(b)

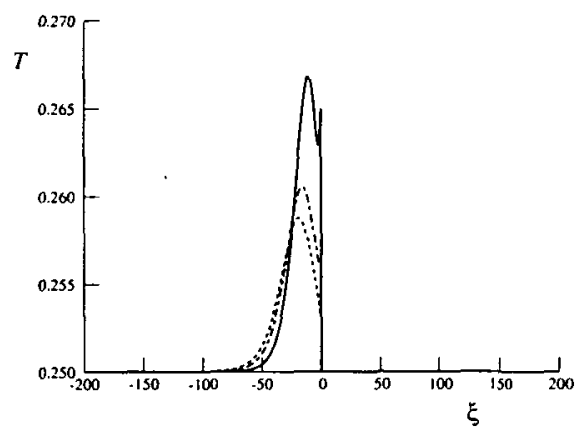

(d)

FIGURE 2. Wave profiles with fitted shocks, for $\beta=0.9, X_{0}=1$ and $\theta_{a}=0.25$, for the three values of shock amplitude $\mathscr{A}_{s}=0.003$ (dotted line), 0.006 (chain-dot line) and 0.015 (solid line). Profiles shown are for (a) gas velocity, (b) fuel concentration, (c) gas density and (d) gas temperature.

a solid line. These solutions were obtained with up to one million numerical integration points downstream of the shock, and may therefore be regarded as effectively exact.

The gas velocity shown in Figure 2(a) is zero ahead of the the shock, but rises discontinuously to the value given by (7.17) immediately behind the shock, located at $\xi=0$. For small amplitude $\mathscr{A}_{s}$ the gas velocity $u$ continues to rise monotonically, until it reaches its final value far downstream as $\xi \rightarrow \infty$. However for large shock amplitude, the velocity drops immediately behind the shock before rising again to its downstream value. This can be seen for the profile with shock amplitude $\mathscr{A}_{s}=0.015$ shown in Figure 2(a).

It is interesting to compare the corresponding shock wave profiles for the fuel concentration $X$, shown in Figure 2(b), with the travelling wave profiles of Figure $1(\mathrm{~b})$. For total wave amplitudes $\mathscr{A}_{T}$ that were not sufficiently large to produce a shock, it was seen in Figure 1(b) that the fuel concentration actually increased slightly above its ambient value $X_{0}$, at the wave front, before falling abruptly to the upstream value $X_{0}$. 


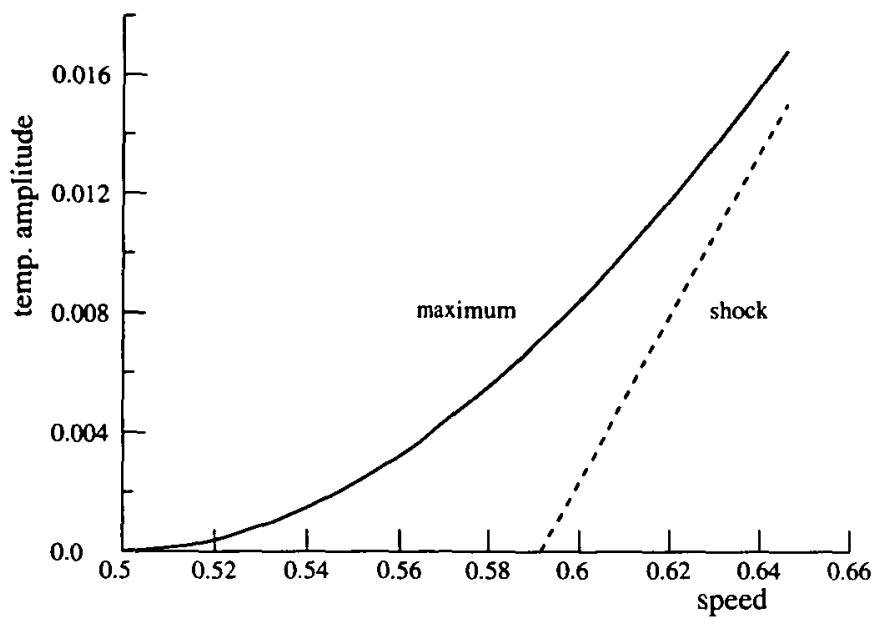

FIGURE 3. Temperature amplitude as a function of wave speed, for the case $\beta=0.9, X_{0}=1$ and $\theta_{a}=0.25$. The solid line is the maximum wave amplitude $\mathscr{A}_{T}$ (given by (5.3) in the absence of shocks, and computed numerically when shocks are present). The dashed line represents the shock wave amplitude $\mathscr{A}_{s}$ (obtained from (7.16)).

This is possible because of the compressibility of the gas in the pipe. This effect is exaggerated by the presence of a shock, as in Figure 2(b), since the shock causes a sharp increase in $X$ which has the appearance of a narrow spike, before the fuel concentration $X$ drops behind the wave as a result of having been consumed in the combustion process.

The gas density profiles in Figure 2(c) have much the same qualitative shapes as for those in Figure 1(c), except of course for the presence of the shock jump at $\xi=0$. A similar observation can be made for the temperature profiles in Figure 2(d). As the amplitude is increased, the temperature peak continues to become more asymmetrical, and for large amplitudes, the shock almost completely dominates the wave front, as can be seen for the solution with $\mathscr{A}_{s}=0.015$.

The results of Figure 1 for amplitudes lower than the value (8.1) at which the shock first forms, and those in Figure 2 for which a shock is present, are unified in Figure 3. This diagram shows how the temperature amplitude of the wave varies with the speed, both for the case of the travelling solitary wave (before a shock is formed) and for the travelling shock wave; the parameters have the same values as in Figures 1 and 2. The curve sketched with a solid line and labelled "maximum" is simply the biggest temperature excursion for the wave of a particular wave speed. It is either given by (5.3) for the case of the travelling solitary wave, or else computed from the numerical solution by taking the difference between the maximum and minimum values of temperature on the profile in the case when a shock is present. It can be seen 


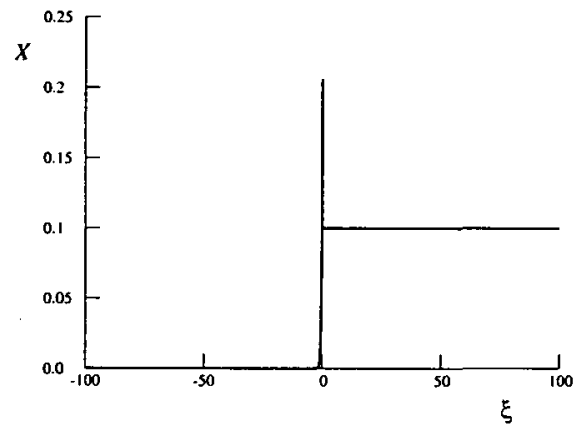

(a)

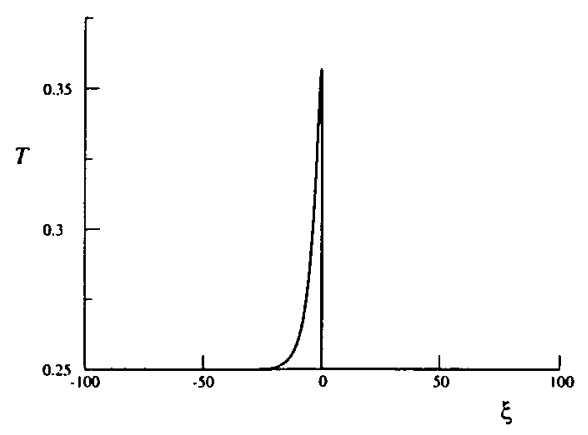

(b)

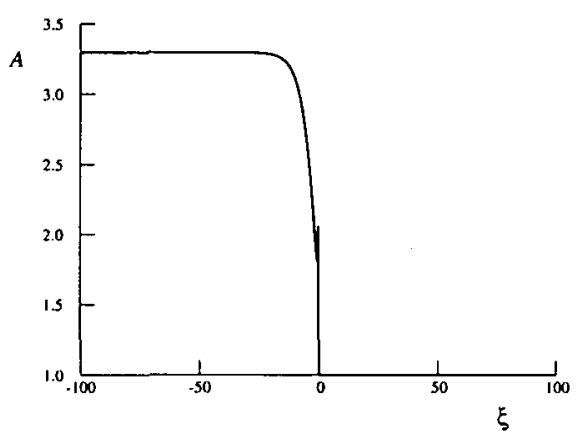

(c)

FiguRE 4. Wave profiles with fitted shocks, for $\beta=9, X_{0}=0.1$ and $\theta_{a}=0.25$. The shock amplitude is $\mathscr{A}_{s}=0.1$. Profiles shown are for (a) fuel concentration, (b) gas temperature and (c) oxygen concentration.

that the curve really is continuous and possesses a continuous first derivative, showing that the solution with a shock present genuinely forms part of the same mathematical branch as the solutions with no shock.

The curve drawn with a dashed line in Figure 3 is the shock amplitude $\mathscr{A}_{s}$ and has been plotted against the wave speed, which is now the shock speed $c_{s}$. This relation is known in closed form, and so the dashed curve is merely a sketch of (7.16). The point at which the shock first appears is given by (8.1).

In a study of combustion in a porous medium, Norbury and Stuart [19] observed that another type of combustion wave ought to be possible, in which the front is so strong that all the fuel behind the wave is used up, so that the flame simply goes out in the region downstream of the wave. We have sought such solutions in this work also, and have confirmed their presence for appropriate parameter values. Figure 4 shows an example of such a wave, in which complete extinction has occurred behind the moving front. For this case, the parameter values were chosen to be $\beta=9, X_{0}=0.1$ with ambient temperature $\theta_{a}=0.25$, and the wave shown has shock amplitude $\mathscr{A}_{s}=0.1$. 
It turns out that the wave speeds are identical for this set of parameters, so that Figure 3 applies to this case also.

The fuel concentration $X$ is displayed in Figure 4(a), where it can be seen that the shock results in a large narrow spike of concentration near $\xi=0$, followed by total exhaustion of the fuel. However, it must be pointed out that the solution shown here is part of the same mathematical branch as the other solution types presented in this paper, and so does not represent any type of qualitatively new phenomenon. The temperature profile for this wave is shown in Figure 4(b), and has now become quite asymmetrical, with the strong shock dominating the entire reaction front. Figure 4(c) shows the oxygen concentration $A$ for this wave; in the previous results (Figures 1 and 2) this quantity was not discussed since it was identical to the fuel concentration $X$ by virtue of the fact that $X_{0}$ had the value one there. In Figures 4 , however, the choice of ambient fuel concentration $X_{0}=0.1$ is responsible for the difference between the two profiles for the quantities $X$ and $A$. The rapid consumption of the fuel $\mathrm{X}$ at the wave front is responsible for a small decrease in the oxygen concentration $A$ immediately behind the wave, but after all the fuel is exhausted, the strong compression caused by the moving shock ultimately causes the oxygen concentration $A$ to rise far downstream.

\section{Summary and conclusions}

This paper has presented a fairly complete model for combustion waves in a compressible gas, with a standard second-order chemical reaction occurring. Admittedly, we have not included the effects of thermal diffusion or gas viscosity in this model, but it seems unlikely these effects would cause much change to the overall solution patterns shown here. This is supported by the work of Clarke [3], who demonstrated that the effects of thermal diffusion become weak at higher Mach numbers. Of course, diffusive and viscous effects would smooth a discontinuous shock into a continuous front, but this represents only a minor change to the solution.

For waves of small to moderate temperature amplitude, a weakly non-linear analysis shows that the temperature profile has the classical "sech squared" profile of a soliton (see Whitham [23], for example). As the wave amplitude is increased, these symmetrical soliton profiles begin to lose their symmetry, and steepen at the wave front. Eventually a shock is formed there, when the propagation speed $c_{s}$ becomes equal to the speed of sound $\sqrt{\gamma \theta_{a}}$. If the wave amplitude is sufficiently large, the shock can result in the complete exhaustion of the fuel downstream.

It is a remarkable feature of this model that the governing equations can actually be reduced to a phase plane system. However, many of the usual advantages of phase plane methods seem not to be available here, principally because the steady states in 
the mathematical $(u, X)$ phase plane do not occur as isolated points, but rather as two vertical lines at $u=0$ and $u=c-\theta_{a} / c$. Thus, although it is possible to classify the behaviour near the steady states in the usual way, there appears little point in doing so, since the actual points in the phase plane corresponding to the beginning and end of the wave are determined by external parameters such as the ambient concentration $X_{0}$. This is further complicated by the presence of shocks in the model.

Nevertheless, a novel and very powerful shooting method has been developed for the travelling wave problem, and is capable of such high accuracy that its results may be regarded as essentially exact. When shocks are present in the profile, the method has been modified to fit them exactly.

The results show a smooth transition from a subsonic wave to the supersonic case in which a shock is also present in the profile. The two different situations are seen to be part of the same solution branch, as is evident from Figure 3. This smooth transition is essentially a consequence of the heat loss to ambient, included in the energy equation (2.8). Although this Newtonian cooling term is normally only of significance for fluid particles close to the walls of the pipe, in practice the fluid contact with the walls is likely to be enhanced by the effects of turbulence, so justifying the inclusion of this term in the energy equation.

The usual Arrhenius rate law (2.9) is clearly inappropriate to this problem over all temperature values, since it would predict that gas burning would always occur; this is the 'cold boundary' difficulty of combustion theory. We have replaced this law with a type of caricature function (2.14) which has the same qualitative profile, except that it switches off below some ignition temperature, taken here to be the ambient temperature $\theta_{a}$ simply for ease of analysis. Other functions can be tried in this law and appear not to have much effect upon the results, although some finite value for the derivative $\beta=k^{\prime}\left(\theta_{a}\right)$ is needed for the weakly non-linear analysis of Section 4 to apply. It ought to be possible to investigate the effects of alternative chemistry to the scheme (2.1) within a similar mathematical framework, and this awaits future work.

\section{References}

[1] H. Bi and A. K. Agrawal, "Study of autoignition of natural gas in diesel environments using computational fluid dynamics with detailed chemical kinetics", Combust. Flame 113 (1998) 289302.

[2] J. D. Buckmaster, The mathematics of combustion, Frontiers in Appl. Math. (SIAM, Philadelphia, 1985).

[3] J. F. Clarke, "On changes in the structure of steady plane flames as their speed increases", Combust. Flame 50 (1983) 125-138.

[4] D. P. Coppersthwaite, J. F. Griffiths and B. F. Gray, "Oscillations in the $\mathrm{H}_{2}+\mathrm{Cl}_{2}$ reaction: Experimental measurements and numerical simulation", J. Phys. Chem. 95 (1991) 6961-6967. 
[5] L. K. Forbes, "Limit-cycle behaviour in a model chemical reaction: the Sal'nikov thermokinetic oscillator", Proc. Roy. Soc. London Ser. A 430 (1990) 641-651.

[6] L. K. Forbes, "One-dimensional pattern formation in a model of burning", J. Austral. Math. Soc. Ser. B 35 (1993) 145-173.

[7] L. K. Forbes, "Stationary circular target patterns in a surface burning reaction", J. Engin. Math. 30 (1996) 471-486.

[8] L. K. Forbes, "A two-dimensional model for large-scale bushfire spread", J. Austral. Math. Soc. Ser. B 39 (1997) 171-194.

[9] L. K. Forbes, M. R. Myerscough and B. F. Gray, "On the presence of limit-cycles in a model exothermic chemical reaction: Sal'nikov's oscillator with two temperature-dependent reaction rates", Proc. Roy. Soc. London Ser. A 435 (1991) 591-604.

[10] B. F. Gray and L. K. Forbes, "Analysis of chemical kinetic systems over the entire parameter space IV. The Sal'nikov oscillator with two temperature-dependent reaction rates", Proc. Roy. Soc. London Ser. A 443 (1994) 621-642.

[11] B. F. Gray and M. J. Roberts, "An asymptotic analysis of the Sal'nikov thermokinetic oscillator", Proc. Roy. Soc. London Ser. A 416 (1988) 425-441.

[12] P. Gray and J. F. Griffiths, "Thermokinetic combustion oscillations as an alternative to thermal explosion", Combust. Flame 78 (1989) 87-98.

[13] P. Gray and W. Kordylewski, "Travelling waves in exothermic systems", Proc. Roy. Soc. London Ser. A 416 (1988) 103-113.

[14] P. Gray and S. K. Scott, Chemical oscillations and instabilities. Non-linear chemical kinetics (Clarendon Press, Oxford, 1990).

[15] J. F. Griffiths, "The fundamentals of spontaneous ignition of gaseous hydrocarbons and related organic compounds", Adv. Chem. Phys. 64 (1986) 203-304.

[16] S. R. Kay and S. K. Scott, "Oscillations of simple exothermic reactions in a closed system II. Exact Arrhenius kinetics", Proc. Roy. Soc. London Ser. A 416 (1988) 343-359.

[17] H. W. Liepmann and A. Roshko, Elements of gasdynamics (John Wiley and Sons, New York, 1957).

[18] J. D. Murray, Mathematical biology, Biomathematics Texts 19 (Springer, Berlin, 1989).

[19] J. Norbury and A. M. Stuart, "Travelling combustion waves in a porous medium. Part I-Existence", SIAM J. Appl. Math. 48 (1988) 155-169.

[20] I. Ye. Sal'nikov, "Contribution to the theory of the periodic homogeneous chemical reactions", $Z h$. Fiz. Khim. 23 (1949) 258-272.

[21] K. K. Tam, "Traveling wave solutions for a combustion problem", Stud. Appl. Math. 81 (1989) 117-124.

[22] R. O. Weber, G. N. Mercer, H. S. Sidhu and B. F. Gray, "Combustion waves for gases ( $L e=1)$ and solids $($ Le $\rightarrow \infty)$ ", Proc. Roy. Soc. London Ser. A 453 (1997) 1105-1118.

[23] G. B. Whitham, Linear and nonlinear waves (Wiley, New York, 1974).

[24] F. A. Williams, Combustion theory (Addison-Wesley, Massachusetts, 1965). 